\title{
Vom Kolonialsoldaten zum Staatsbürger. Ein Zivilporträt westafrikanischer Veteranen der französischen Armee
}

(vorgetragen in der Plenarsitzung am 4. Dezember 2009)

Herannahende Jahrestage setzen gewöhnlich Impulse, sich mit einem historischen Sachverhalt erneut zu beschäftigen. Dies hat auch mich inspiriert, Ihnen heute eine Thematik aus meiner nunmehr zehn Jahre zurückliegenden Forschung über burkinische Veteranen des Zweiten Weltkriegs zu präsentieren. Der bevorstehende Jahrestag, um den es mir dabei zu tun ist, bezieht sich auf den von den einen als Entlassung in die Unabhängigkeit bezeichneten, von den anderen als Erringung der staatlichen Souveränität gefeierten historischen Moment des Eintritts der meisten subsaharischen Kolonien in die internationale Staaten-

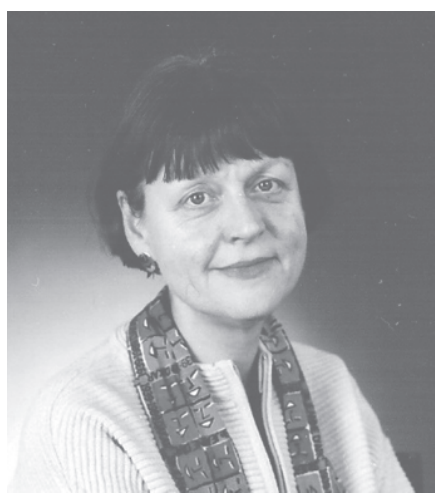

Brigitte Reinwald, Professorin für Afrikanische Geschichte an der Leibniz-Universität Hannover, O. Mitglied der Akademie seit 2009 gemeinschaft. Dieser Moment jährt sich in 2010 zum 50. Mal und ist gegenwärtig Ausgangspunkt für eine kritische Bilanzierung dieses letzten halben Jahrhunderts postkolonialer Entwicklung Afrikas, die sich u.a. in einer Reihe geplanter Konferenzen und Publikationen niederschlagen wird. Mir bietet er heute Anlass für eine kritische Beleuchtung des angelegten Periodenmodells, demzufolge das Jahr 1960 als Ausdruck eines historischen Paradigmenwechsels für afrikanische Gesellschaften gilt und für deren Aufbruch in eine neue Ära steht. Mein Argument, das ich Ihnen am Beispiel ehemaliger westafrikanischer Kolonialsoldaten der französischen Armee näher erläutern möchte, ist vielmehr, dass die hier postulierte Zäsur, zieht man die Erfahrungsperspektiven afrikanischer Bevölkerungs- 
gruppen in Betracht, bereits ab 1944/45 einsetzte und gewissermaßen auch einen Auftakt für Zukunftsprojekte sozialer und politischer Natur darstellte, denen 1960 bereits in erheblichem Umfang die Flügel gestutzt waren. Oder wie es der Afrikahistoriker Frederick Cooper formuliert: Forderungen und Vorstellungen, die Afrikanerinnen und Afrikaner in der Dekade von 1945 bis 1955 hinsichtlich ihrer eigenen Zukunft entwickelten, waren nicht nur nicht deckungsgleich mit dem, was sie ab 1960 dann erhielten, sondern sie überraschen uns Historikerinnen und Historiker auch dahingehend, was bei näherer Betrachtung der spätkolonialen Verhältnisse in jener Dekade noch alles möglich schien (Cooper 1997).

Welche Bedeutung Angehörige westafrikanischer Gesellschaften dem Zeitraum der späten 1940er Jahre im Rückblick beimessen, mag folgende recht bündige Antwort verdeutlichen, die ich von einem ehemaligen burkinischen Oberfeldwebel auf meine Frage erhielt, wie er jene Jahre erlebt habe. Sie sei hier stellvertretend für eine Reihe ähnlich lautender Bekundungen zitiert:

C'était le début de l'indépendance. Quand l'indépendance a déclenché, alors beaucoup veut [sic!] être libre, avoir la liberté. Parce que, il y avait des travaux forcés. [... ] Mais on était fatigué! Parce que les gens, on les mettait de côté [... ] on les envoie aux travaux forcés sans, sans faire, sans salaires et tout ça là. Voyez, c'était dangereux. Alors donc, quand la guerre d'indépendance a éclaté, tout le monde était fier de ça. [...]

(Das war der Beginn der Unabhängigkeit. Also, was die Unabhängigkeit ausgelöst hat, war, dass viele frei sein wollten, die Freiheit haben wollten. Denn es gab die Zwangsarbeit. [... ] Aber wir hatten genug davon! Denn man nahm die Leute einfach zur Seite [...] schickte sie in die Zwangsarbeit, ohne alles, ohne Lohn und all das. Sehen Sie, das war gefährlich. Als dann also der Unabhängigkeitskrieg ausbrach, waren alle stolz darauf. [... ]. B.S., Bobo-Dioulasso 10.3.1999, Übersetzung B.R.)

Erstaunlich ist hier nicht in erster Linie, dass jener Veteran Erfahrungen der kolonialen Zwangsarbeit ins Gedächtnis ruft, die im April 1946 durch eine Gesetzesinitiative des ivorischen Abgeordneten Félix Houphouët-Boigny vom französischen Parlament abgeschafft wurde. Diese Bezugnahme lag, wie ich einen Moment später erfahren sollte, zum einen auch darin begründet, dass er selbst noch für die französische Armee zwangsrekrutiert worden war. Zum anderen hatten sich die Veteranen des Zweiten Weltkriegs in überwältigender Mehrheit, aber auch aktive Soldaten im Südwesten Obervoltas seinerzeit an den Massendemonstrationen beteiligt, die jene Initiative flankierten. Dass der Interviewte jenen Protesten jedoch die Bedeutung eines Unabhängigkeitskrieges beimaß, den es weder zum damaligen noch zu einem späteren Zeitpunkt in seiner Heimatkolonie Obervolta je gegeben hat, signalisiert, dass für ihn wie für viele andere, Zivilpersonen wie Mili- 
tärs, die Freisetzung vom Arbeitszwang den entscheidenden Wendepunkt in seinem Leben markierte, dem gegenüber die spätere staatliche Unabhängigkeit stark in den Hintergrund trat. Eine Reihe anderer Interviewpartner merkten in diesem Zusammenhang an, 1945 habe das Zeitalter der politique die Ära der Kolonisation abgelöst, was man indes als mehrdeutige Äußerung interpretieren sollte. Verbanden dies nämlich die einen im positiven Sinne damit, dass anstelle des Gehorchens die Zeichen von da an auf Verhandeln standen, so brachten andere hierin eher ihr Missfallen darüber zum Ausdruck, dass nunmehr die Fähigkeit, mit doppelter Zunge zu sprechen, dem alten Ehrenwort den Rang abgelaufen hatte und die Menschen nun denjenigen anhingen, die ihnen die meisten Versprechungen machten. Andere Interviewte wiederum erwähnten schließlich das Stichdatum 1960 überhaupt nicht bzw. quittierten meine Fragen danach allenfalls mit einem lakonischen Halbsatz, in dem selbstredend auch ihre Enttäuschungen über die mediokre Bilanz von fast vierzig Jahren staatlicher Unabhängigkeit mitschwangen.

Daraus lässt sich nun die Feststellung ableiten, dass jene Männer das Kriegsende bzw. die erste Dekade nach 1945 nicht nur als Periode politischen Umbruchs und Aufbruchs ins Neue erinnerten, sondern sich seinerzeit auch, wie eine nähere Betrachtung ihrer postmilitärischen Lebensverläufe bestätigt, als politische, soziale wie kulturelle Akteure gefordert sahen, jene Zeit des Übergangs mitzugestalten. Auf welche Weise sie sich nach der Rückkehr aus dem Krieg in verschiedenen politisch-gesellschaftlichen Kräftefeldern bewegt und betätigt und welche Rolle dabei ihre Erfahrungen im Krieg - d.h. an der Front, in der Garnison, im Kriegsgefangenenlager, bei der Repatriierung und Demobilisation - sowie im spätkolonialen Alltag der 1940er und der 1950er Jahre gespielt haben, war Gegenstand meiner historischen Fallstudie (Reinwald 2005). Wie kam es, so habe ich mich gefragt, dass diesen Männern die Armee gewissermaßen zur Bildungsanstalt, der Krieg zum Lernfeld wurde? Welche Konsequenzen haben sie daraus gezogen? Was war bzw. wurde der ehemaligen Soldaten und künftigen Staatsbürger politisches Vaterland? Wem, wenn überhaupt, fühlten sie sich loyal verbunden?

Hauptsächliches Quellenkorpus für meine historische Gruppenbiographie waren rund vierzig narrative Interviews, die ich 1999 in drei verschiedenen Regionen der Republik Burkina Faso - der Südwestregion um die Stadt Bobo-Dioulasso, der Hauptstadt Ouagadougou sowie im Nordwesten im ruralen Einzugsbereich des Bezirks von Tougan - durchgeführt und aufgezeichnet habe. Meine Auswahl dieser Untersuchungsgebiete war forschungsstrategisch dadurch motiviert, dass es sich jeweils um Regio- 
nen handelte, die durch eine vergleichsweise „hohe Dichte“ an Veteranen gekennzeichnet waren, hinsichtlich ihrer Entwicklungsdynamik post bellum, vor allem in den Punkten politischer Orientierung und sozialer Strukturierung der Zielgruppen (Mobilitäts- und Urbanisierungsgrad), jedoch markante Unterschiede aufwiesen. Auch wenn das so konstituierte Quellenkorpus mir Binnenvergleiche ermöglichte und dazu beigetragen hat, in einem bestimmten Mikromilieu (Dorf, Stadtviertel) erhobene Daten auf Übertragbarkeit zu prüfen, so eignet ihm ein grundsätzliches Problem von Oral History-Untersuchungen. Es wirft nämlich die Frage danach auf, bis zu welchem Grad die Ergebnisse meiner vergleichenden Mikrostudie verallgemeinerbar sind, zumal wir im Falle afrikanischer Mannschaftssoldaten kaum über Primärzeugnisse aus dem Krieg selbst, geschweige denn aus ihrem postmilitärischen Alltag verfügen. Folglich gilt es, hinsichtlich retrospektiv erhobener Lebensberichte immer auch deren Überformung durch spätere Lebenszusammenhänge und d.h. auch: taktisch und strategisch motivierte Rücküberschreibungen früherer Erfahrungen in Betracht zu ziehen (vgl. dazu Reinwald 2005, 30-32).

Warum dieses Augenmerk auf (ehemalige) Soldaten und wieso nun gerade Obervolta/Burkina Faso? Militärdienst und Kriegseinsätze waren ein Massenphänomen, von dem im Verlauf des 20. Jahrhunderts mehrere hunderttausend Männer verschiedener Generationen aus Frankreichs afrikanischen Kolonien betroffen waren, sei es, dass sie zu Beginn des Jahrhunderts als Hilfstruppen für die koloniale Eroberung und „Befriedung“, als Kombattanten in beiden Weltkriegen und den anschließenden Kolonialkriegen in Indochina und Algerien oder auch bis in die 1960er Jahre hinein zur Bekämpfung antikolonialer Bewegungen in Madagaskar, Marokko, Mauretanien, Niger und Kamerun eingesetzt wurden.

\begin{tabular}{|l|l|l|l|}
\hline Erster Weltkrieg & Zweiter Weltkrieg & $\begin{array}{l}\text { Indochinakrieg } \\
(1945-1954)\end{array}$ & $\begin{array}{l}\text { Algerienkrieg } \\
(\mathbf{1 9 5 4 - 1 9 6 2})\end{array}$ \\
\hline 165229 & $\sim 100000$ (1939-40) & $\sim 46800$ & $\sim 15000$ \\
& $\begin{array}{l}\sim 100000(1942-45) \\
\text { (FFL und Alliierte Truppen, } \\
\text { enthält auch }\end{array}$ & & \\
& Franz.-Äquatorialafrika) & & \\
\hline $\begin{array}{l}\text { davon in Europa und } \\
\text { Nordafrika: }\end{array}$ & $\begin{array}{l}\text { davon in Kriegsgefangenschaft: } \\
134077\end{array}$ & & \\
& $\begin{array}{l}\text { (Subsah. Afrika und Madagaskar } \\
\text { gesamt) }\end{array}$ & & \\
\hline davon Tote und Vermisste: & $\begin{array}{l}\text { davon Tote und Vermisste: } \\
24938\end{array}$ & & \\
\hline
\end{tabular}

Abbildung 1: Westafrikanische Truppenkontingente Frankreichs im 20. Jahrhundert (Zahlenangaben und Schätzungen nach Reinwald 2005, 36-41) 
Die hier aufgelisteten Zahlen sollen lediglich eine grobe Größenordnung indizieren, was den Gesamtumfang der kämpfenden Einheiten betrifft. Welche Signifikanz Rekrutierungen und Kriegseinsätze für die einzelnen Kolonien hatten, lässt sich nur unter Berücksichtigung der jeweiligen demographischen Ausgangslage sowie kolonialpolitischer Zielsetzungen ermitteln. Im Ergebnis lieferten nämlich rohstoffarme und enklavierte Hinterlandkolonien wie Obervolta, aber auch Soudan (heute Mali) und Guinea in allen erwähnten Kriegen einen ungleich höheren Anteil an Soldaten als so genannte prosperierende Kolonien wie etwa Senegal, der Süden der Elfenbeinküste oder Dahomey. Deshalb ist auch der Sammelbegriff Tirailleurs Sénégalais, d.h. Senegalschützen, wie man die westafrikanischen Einheiten der Kolonialinfanterie gemeinhin bezeichnete, irreführend, insofern er die ausschlaggebende Bedeutung der Hinterlandkolonien als Reservoir für militärische und zivile Arbeitskraft okkultiert.

Als zweiter Aspekt sollte auch der enge Zusammenhang zwischen militärischer und ziviler Zwangsarbeit beachtet werden: Entgegen landläufigen Annahmen handelte es sich bei den afrikanischen Soldaten der französischen Armee in der Regel nicht um Freiwillige oder gar Söldner. Aushebung und Rekrutierung für die Kolonialinfanterie basierten auf der 1910 verordneten dreijährigen Wehrpflicht für alle männlichen Kolonialuntertanen über 20 Jahren und ordneten sich somit in ein weit verzweigtes System der Zwänge ein, das durch militärische, aber auch durch zivile Zwangsarbeit und Steuerabgaben von Männern, Frauen und Kindern - und somit Angehörigen aller Altersgruppen ab dem Alter von zehn Jahren - gekennzeichnet war. Vor diesem Hintergrund erklärt sich auch die eingangs zitierte euphorische Bekundung des Oberfeldwebels hinsichtlich der Abschaffung der Zwangsarbeit, zumal er hier als Zeitzeuge herangezogen werden kann, was die Auswirkungen jener flächendeckenden und lang andauernden repressiven Inanspruchnahme indigener Arbeitskraft in den Hinterlandkolonien betrifft.

Fragt man also nach der Auswirkung der Weltkriege auf Lebensverläufe und Handlungsstrategien westafrikanischer Bevölkerungen, dann muss man diese Kriege in einem zeitlichen und räumlichen Horizont betrachten, der sich von etwa 1910 bis zum Ende der 1950er Jahre erstreckt und durch die intensive Mobilisierung für das Kolonialwerk und eine daraus resultierende Mobilität breiter Bevölkerungsgruppen geprägt war, die sich vor allem in Westafrika selbst entfaltete. Den Kriegen kam in diesem Zusammenhang zwar eine Katalysatorfunktion zu, sie waren aber, was das Erfahrungsspektrum von Afrikanerinnen und Afrikanern angeht, kolonial konnotiert. 
Frederick Cooper zufolge hat sich in jenem Zeitraum die Auseinandersetzung afrikanischer Gesellschaften mit dem „hegemonialen Projekt europäischer Kolonisation" (Cooper 1997, 409) nicht nur intensiviert, sondern sie erfasste erstmals auch breite Teile der Bevölkerung dahingehend, dass diese sich ihrer Rolle im Räderwerk des kolonialen Gefüges bewusst wurden und daraus schließlich Forderungen nach Gleichbehandlung ableiteten. Für die Gruppe der Soldaten kann ich ergänzen, dass solche Bewusstwerdungsprozesse überwiegend von ihren Bewegungen und Erfahrungen jenseits des engen Rahmens der Heimatkolonie angestoßen wurden. Im Verlauf ihrer kriegsbedingten Itinerarien sind sie sich des Spannungsverhältnisses zwischen der kolonialen Situation, aus der sie aufgebrochen waren, und einer möglichen Zukunft als Gleiche überhaupt erst bewusst geworden. Ihre sich wandelnden Selbst- und Fremdwahrnehmungen mündeten jedoch nicht, wie von der älteren Forschung postuliert, zwangsläufig und dauerhaft in antikoloniale oder nationale Handlungsorientierungen ein, sondern schlugen sich vielfach in unspektakulären, in Wirkung und Nachhaltigkeit deswegen doch nicht weniger markanten Aktivitäten der Kriegsheimkehrer nieder. In meiner Studie habe ich diesbezüglich drei Wirkungsfelder unterschieden: das unmittelbare familiäre und dörfliche Milieu, das weitere soziale Beziehungsfeld wie z.B. Veteranenverbände, Berufsgruppen, Freundschaftskreise, Glaubensgemeinschaften und schließlich das politische Kräftefeld. Mit einem Beispiel aus dem familiären Milieu möchte ich Ihnen ein solches Wirkungsfeld exemplarisch illustrieren.

Um Ihnen zumindest ein grobes Soziogramm der Veteranen zu geben: die große Mehrheit der Kriegsheimkehrer war nach meiner Einschätzung nicht von einer Mission beseelt, d.h. sie wollten nicht per se als soziale und kulturelle Modernisierer oder gar antikoloniale Akteure wirken, sondern arbeiteten gewissermaßen auf eigene Rechnung, um sich in ihrem Milieu wieder zurechtzufinden oder einen Statuszuwachs zu erringen, den sie ihrem durch Krieg und Kriegsdienst veränderten Selbstbild für angemessen hielten. Dabei prägten sie einen spezifischen Lebensstil des ancien combattant, in dem sich das Grenzgängertum spiegelte, das sie gewiss zum Ausdruck bringen wollten, in das sie jedoch auch von ihrer Umgebung immer wieder hineingedrängt wurden. Als Einzige ihrer näheren oder weiteren Umgebung, die in Europa gewesen waren, markierten sie ihre Weltläufigkeit durch bestimmte Konsumpraktiken, Siedlungsformen und Zukunftsprojekte. Für ihre Familien und Dorfnachbarn wurden sie dadurch jedoch in der Regel zu internal strangers, die man nicht so ohne weiteres reintegrierte, wie dies etwa auch die Ethnologin Heike Behrend (1999, 25) im Hinblick auf postmilitärische Lebensverläufe ugandischer Ex-Soldaten der 


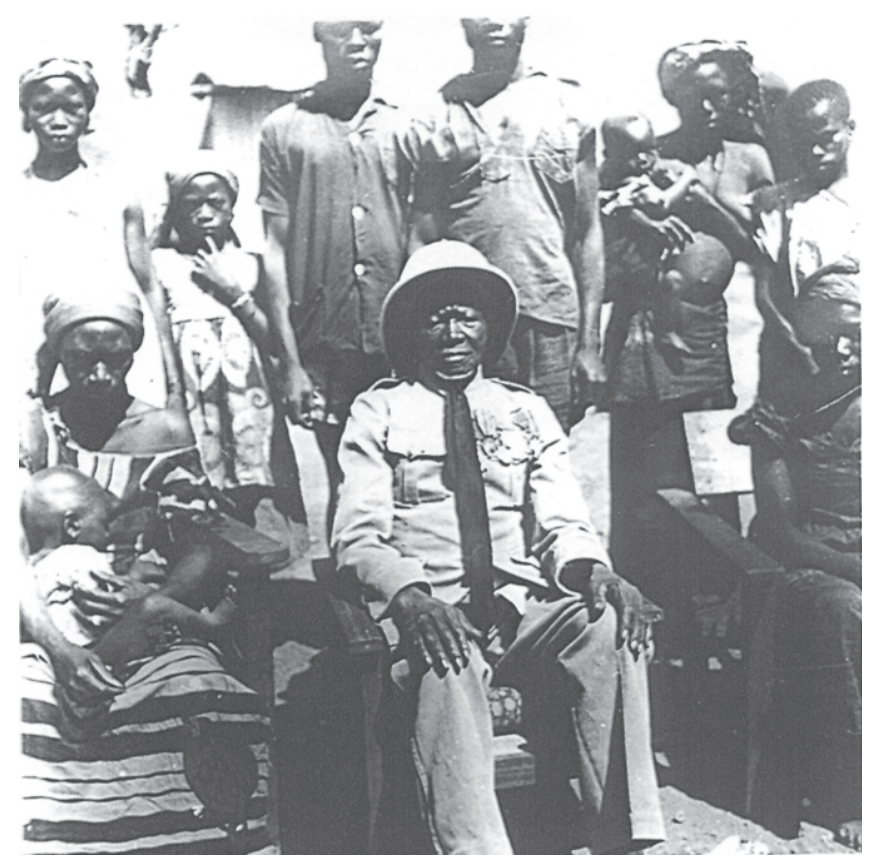

Abbildung 2: Kriegsveteran im Kreise seiner Familie. (Fotografie Cissé, Dakar/Senegal, undatiert, um 1955, Abdruck mit freundlicher Genehmigung des Institut Fondamental d'Afrique Noire, IFAN, Dakar)

1980er Jahre festgestellt hat. Dass viele Veteranen vom Dorf in die Stadt umzogen oder aber in einer Art Überanpassung an traditionelle Normen versuchten, neue Allianzen zu schmieden, indem sie sich z.B. polygam verheirateten oder Chefämter anstrebten, interpretiere ich in erster Linie auch als Reaktion auf diese gegenseitige Entfremdung.

In der hier präsentierten Fotografie - einem Fundstück aus den Dakarer Archiven, aufdem mit großer Wahrscheinlichkeit allerdings kein voltaischer Veteran sondern ein senegalesischer Kombattant des Zweiten Weltkriegs in die Kamera blickt - drückt sich nach meiner Einschätzung dieses Grenzgängertum sehr gut aus. Wohl eine Auftragsarbeit eines örtlichen Fotografen, symbolisiert dieses Porträt, wie sich der Veteran mit seinen Orden, seiner US-amerikanischen Ausgehuniform und seinem Tropenhelm einerseits als Angehöriger der alliierten Truppen inszeniert, andererseits jedoch darum bemüht ist, seine erfolgreiche Rückbindung und Familiengründung zu demonstrieren, indem er sich, wie anzunehmen ist, von seinen beiden Ehefrauen und Kindern aus diesen Verbindungen einrahmen lässt. 
Jenseits der Symbolik möchte ich jedoch auf eine markante Neuerung hinweisen, die auf die Veteranen des Zweiten Weltkriegs zurückzuführen ist und sich mittelfristig insofern nachhaltig auswirkte, als sie die Entstehung einer bildungsorientierten westafrikanischen Mittelschicht mitbefördert hat: den Impetus der ehemaligen Soldaten zur Einschulung ihrer Kinder, Jungen wie Mädchen gleichermaßen. Die Signifikanz, die sie dem Schulbesuch beimaßen, lässt sich über die Ergebnisse meiner Untersuchung hinaus als genereller, Regionen übergreifender Trend festhalten. Aus einer ganzen Reihe ähnlich lautender Bekundungen sei zur Illustration lediglich folgendes Statement des uns schon bekannten Oberfeldwebels herangezogen:

Mais nous qui ont [sic!] fait l'armée, quand même, on a vu l'extérieur quand même. Un ancien combattant qui n'envoie pas ses enfants à l'école, bien lui, il n'a rien vu, il n'a rien suivi. [...] si tu ne mets pas ton enfant à l'école et il arrive à un certain âge et s'il n'arrive pas à parler français [...] on ne sait pas ce qu'il va devenir. Nous qui avons été en France, on connaît tous ces problèmes.

(Aber immerhin haben wir, die wir in der Armee waren, ja doch etwas von der Welt gesehen. Ein Veteran, der dann seine Kinder nicht in die Schule schickt, also der, der hat nichts gesehen, der hat nichts gelernt. [...] Wenn du dein Kind nicht zur Schule schickst und es erreicht ein gewisses Alter und kann kein Französisch [...] bei dem weiß man nicht, was aus ihm werden soll. Wir, die wir in Frankreich waren, wir kennen alle diese Probleme.) B.S., Bobo-Dioulasso 10.3.1999, Übersetzung B.R.)

Wie Sie sehen, taucht auch hier wieder die Metapher der Armee als Lernfeld auf bzw. motiviert dieser Veteran sein Engagement für die Bildung mit seinen Erfahrungen in Armee und Krieg. Aufschlussreich sind diesbezüglich weitere von den Veteranen angeführte Beweggründe für den Schulbesuch, so etwa, sie hätten damit ihren Kindern Chancen eröffnen wollen, die sie selbst nicht gehabt hatten, oder beabsichtigt, sie dem dörflichen Milieu zu entziehen, das sie für unvereinbar mit ihren Ansichten und Ambitionen hielten. Nimmt man nun alle Bedingungs- und Dynamisierungsfaktoren postmilitärischer Lebensverläufe ehemaliger voltaischer Weltkriegskombattanten zusammen - Umzug in die Stadt, Herauslösung aus dörflichen gerontokratischen Verhältnissen, neolokale Familiengründung und Investitionen in Bildung -, dann lassen sich jene Veteranen als Akteure einer Modernisierung charakterisieren, welche in zugegebenermaßen recht eigenwilliger Weise den politischen und sozialen Transitionsprozess ihrer Gesellschaft im spätkolonialen Kontext von unten und von innen heraus mitgestaltet haben.

Im Bildungsimpetus der Veteranen verkörpert sich also eine von mehreren markanten Facetten des zivilgesellschaftlichen Engagements dieser 
Gruppe, dessen Umsetzung - und das erscheint mir sehr wichtig - in ihrer Reichweite lag und an dem sie stringent und ausdauernd festhielten. Damit jedoch haben sie eine nachhaltige Investition in die Zukunft getätigt, die sich nicht zuletzt auch mit Blick auf ihre zahlreiche Nachkommenschaft keineswegs als Nebensache erwiesen hat. An diesem Wirkungsstrang zeigt sich auch, dass ihr Engagement von ihrem aus Erfahrung gewonnenen performativen Wissen motiviert war, dessen Potential sich vorrangig im kleinen Rahmen entfaltete. Darum haben sie jedoch nicht weniger, um abschließend auf den Titel meines Vortrags zurückzukommen, in staatsbürgerlicher Absicht und Verantwortung gehandelt. Mehr noch: im Gegensatz zu ihren im politischen Kräftefeld der Spätkolonie entfalteten Aktivitäten, mit denen die ehemaligen Soldaten ihre Forderungen nach Teilhabe, Gleichstellung und Freisetzung zum Ausdruck brachten, dabei jedoch von wechselhaften politischen Konjunkturen und nicht zuletzt von ihren eigenen ambivalenten Loyalitätsmustern immer wieder überrumpelt wurden, hat dieses Engagement im Kleinen, multipliziert durch die hohe Anzahl derjenigen, die sich ihm verpflichtet fühlten, sie und ihre Nachkommen immerhin in die Lage versetzt, ihre „durch Unsicherheiten und Widersprüche geprägte Praxis besser zu meistern." (Hörning 1999, 100).

\section{Literatur}

Behrend, Heike 1999. Power to Heal, Power to Kill. Spirit Possession and War in Northern Uganda (1986-1994). In: Spirit Possession. Modernity and Power in Africa (Hg.innen) Heike Behrend \& Ute Luig. London: James Currey \& Kampala: Fountain Publishers et al., S. 20-33.

Cooper, Frederick 1997: The Dialectics of Decolonization: Nationalism and Labor Movements in Postwar French Africa. In: Tensions of Empire. Colonial Cultures in a Bourgeois World (eds.) Frederick Cooper \& Ann Laura Stoler. Berkeley \& Los Angeles \& London: University of California Press, S. 406-435.

Hörning, Karl H. 1999: Kulturelle Kollisionen. Die Soziologie vor neuen Aufgaben. In: Widerspenstige Kulturen. CulturalStudies als Herausforderung (Hg.) Karl H. Hörning \& Rainer Winter. Frankfurt am Main: Suhrkamp Verlag, S. 84-116. (suhrkamp taschenbuch wissenschaft 1423).

Reinwald, Brigitte 2005: Reisen durch den Krieg. Erfahrungen und Lebensstrategien westafrikanischer Veteranen der französischen Kolonialarmee. Berlin: Klaus Schwarz Verlag (Zentrum Moderner Orient, Studien 18). 